\title{
Training Diagnosis for a load Carriage Task
}

\author{
Alun G. Williams, ${ }^{1,2}$ Mark P. Rayson, ${ }^{2}$ AND David A. Jones ${ }^{2,3}$ \\ ${ }^{1}$ Department of Exercise and Sport Science, Manchester Metropolitan University, Alsager, UK; ${ }^{2}$ Optimal \\ Performance Limited, Farnham, UK, ${ }^{3}$ School of Sport and Exercise Sciences, University of Birmingham, \\ Birmingham, UK
}

\begin{abstract}
Williams, A.G., M.P. Rayson, and D.A. Jones. Training diagnosis for a load carriage task. J. Strength Cond. Res. 18(1):30-34. 2004. - To explore the possibility of training diagnosis for a $3.2-\mathrm{km}$ loaded march with a $25 \mathrm{~kg}$ load, 50 men trained for 10 weeks using either running, marching, and endurance-based circuit training (Circuits), or running, marching, and resistance training (Resistance). The march was performed before and after training, and other measurements related to loaded marching were conducted before training only. Each group was ranked by improvement in the loaded march, and divided into significantly different subgroups of 'good' and 'poor' responders (improvements of approximately $20 \%$ vs. $10 \%$ ). For Circuits, there were significant differences between good and poor responders to training in the pretraining ratios of shuttle run: isometric lift strength $(p=0.031)$ and shuttle run: isometric back extension strength $(p=0.033)$. Stronger subjects with lower endurance responded better to Circuits. Resistance tended to show the opposite effect $(p<0.2)$. These are the first objective data on which to prescribe training for load carriage on an individual basis.
\end{abstract}

KEY WoRDS. strength, endurance, loaded marching, training prescription, military personnel

\section{INTRODUCTION}

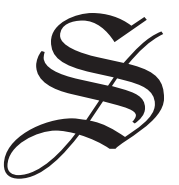

trength diagnosis is a scientific approach to strength assessment and training prescription for a specified strength or power event $(10,11)$. Strength diagnosis involves the use of pretraining measures, which may or may not include the athletic event itself, to determine objectively which type of training is most appropriate for an individual to improve their athletic performance. The prescribed strength training is then focused on the specific area or areas of relative deficiency that an athlete may demonstrate. This approach to practical training problems is probably widely used, and the logical approach has an intuitive appeal to those seeking some objective data on which to base their training programs. However, there is a distinct lack of research that evaluates the efficacy of this strength diagnosis approach.

Initially, authors suggested the use of isometric and eccentric strength tests to determine the "strength deficit" and "voluntary activation capacity" of an athlete in a specific muscle group $(10,11)$. Theoretically, training focused on either hypertrophy or neural adaptation could therefore be prescribed. However, these initial reports did not present any original experimental data $(10,11)$. Only 1 study has used the strength diagnosis approach and presented useful new data (14). Wilson and Murphy (14) used a 6 -second cycle ergometer sprint as their athletic performance of primary interest. Untrained men performed either resistance training or plyometric training for 10 weeks. Subjects with lower pretraining maximum force production during an isometric squat improved cycle performance more than stronger subjects over the course of the resistance training. Presumably, resistance training was particularly appropriate for the weaker subjects, as this training addressed their specific area of relative deficiency. Stronger subjects also tended to respond better to plyometric training. Presumably, an increase in the rate of force development relative to the maximum force was particularly useful for the stronger subjects, as this training addressed their specific area of relative deficiency.

This approach of training diagnosis and subsequent training prescription is not necessarily limited to the physiology of muscle strength. Any athletic performance that can be separated into 2 dichotomous variables that each represents a distinct component of conditioning important for that performance is a suitable candidate for training diagnosis. For example, an athletic event that relies upon both good aerobic power and good muscle strength would be a suitable candidate for training diagnosis.

Loaded march performance (load carriage) is an important duty of military personnel, and the optimal training to improve performance appears to be a combination of resistance training and lower body endurance training $(4,5)$. However, for each individual, the specific area of relative deficiency may be in either muscle strength or aerobic endurance. The basic training program (induction course) used by the British Army has, until very recently, included endurance training but no resistance training (12). Perhaps a more effective approach to military training (at least, to improve loaded march ability) would be to prescribe a training program focused on either resistance or endurance training, based on each individual's pretraining performance in tests of the discrete qualities of strength and endurance. Thus, the purpose of this investigation was to explore the possibility of training diagnosis for loaded marching. Simple pretraining tests of strength and endurance were examined for their ability to discriminate between subjects for whom training focused on either strength or endurance would be most appropriate to improve loaded march performance.

\section{Methods}

\section{Experimental Approach to the Problem}

The time to complete a 2-mile $(3.2-\mathrm{km})$ loaded march with a $25 \mathrm{~kg}$ load was used as the criterion loaded march performance, as this has been identified as a key task required of many British Army personnel (9). Pretraining tests of muscle strength and aerobic endurance thought likely to be related to loaded march performance were used in an attempt to discriminate between those individuals who responded well to resistance training and endurance training and those who did not. 
TABLE 1. Mean $\pm S D$ descriptive characteristics of subjects.

\begin{tabular}{lccc}
\hline & $\begin{array}{c}\text { Circuits } \\
(n=30)\end{array}$ & $\begin{array}{c}\text { Resistance } \\
(n=20)\end{array}$ & $\begin{array}{c}\text { All subjects } \\
(n=50)\end{array}$ \\
\hline Age (y) & $18.7 \pm 2.5$ & $18.6 \pm 2.1$ & $18.7 \pm 2.4$ \\
Stature (m) & $1.74 \pm 0.06$ & $1.77 \pm 0.07$ & $1.75 \pm 0.06$ \\
Mass (kg) & $70.6 \pm 8.8$ & $74.2 \pm 10.5$ & $72.0 \pm 9.6$ \\
\hline
\end{tabular}

\section{Subjects}

Fifty healthy men volunteered to participate in the study. All subjects were British Army recruits, had undergone routine British Army medical screening, received a detailed briefing, and gave informed consent prior to participation. Thirty subjects were assigned to a group whose training was focused on endurance-based circuit training (Circuits), and 20 subjects assigned to a group whose training was focused on resistance training (Resistance). Subjects were assigned to the Circuits or Resistance groups depending solely on their date of entry into the Army (which differed by no more than 8 weeks between any 2 subjects), and there were no significant differences between groups in terms of age, stature, or body mass. Descriptive characteristics of the subjects are presented in Table 1.

\section{Training}

Subjects in both the Circuits and Resistance groups participated in a 10 -week training program that included 71 periods of 40 minutes devoted to physical conditioning. Some of these training periods were paired to form 80minute periods. Due to their military occupation, all subjects also performed other physical exercise such as prolonged marching with various loads while on military exercise, and many 40- or 80-minute periods of drill that averaged about one 40-minute period per day.

The Circuits training program was the existing the British Army basic training program, and included running, marching, and circuit training. Specifically, the 71 periods of training consisted of: 19 sports, 18 circuit training, 10 agility, 9 swimming, 7 endurance, and 2 box lifting periods. An additional 6 periods were used intermittently to undertake assessments of fitness and skills. The sports periods were typically team ball games in a small area. The endurance training periods were typically continuous running. The circuit training was somewhat variable in content, but was generally endurance-based training, consisting of high-repetition, low-force exercises using all major muscle groups. A typical circuit training session included a number of exercises such as shuttle runs, press-ups, squat thrusts, barbell curls, supported dips from a bench, star jumps, sit-ups, and bench steps. However, the modes of exercise used varied between sessions. Typically, exercise-to-rest periods of approximately 30seconds exercise and 10-seconds rest were used in circuit training until completion of all exercises, followed by a rest period of approximately 60 seconds before the next circuit of exercises. However, the precise exercise and rest intervals used also varied between sessions.

The Resistance training program was a modified British Army physical training program, and included running, marching, and whole-body resistance training. Specifically, the 71 periods of training consisted of 28 resistance training, 15 endurance training, 8 agility, 6 box lifting and carrying, 6 sports, 4 circuit-training, and 4 swimming periods. Typically, the resistance training was

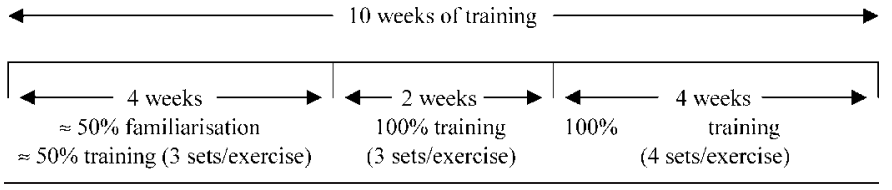

FIGURE 1. Progression in volume of resistance exercise conducted by the Resistance group. Exercises were assisted pull-up, bench press, seated row, dead lift, high curl, leg press, and upright row.

performed twice per week (Figure 1). Approximately $50 \%$ of the resistance training periods in the first 4 weeks were used for familiarization to teach technique and to assess ability on the exercises in order to prescribe individual training loads. Consequently, the volume and intensity of the exercises performed during the first 4 weeks was relatively low. After familiarization, the resistance training consisted of specific exercises at the individually prescribed training loads. Initially, 3 sets of each exercise were performed, progressing to 4 sets per exercise from week 6 . The first set of each exercise was performed at $75 \%$ of the 6 repetition maximum (6RM) training load, while the following sets of each exercise were performed at $100 \%$ of $6 \mathrm{RM}$. Rest periods of 1 minute were used throughout. The resistance exercises were: assisted pullup, bench press, seated row, shoulder press, dead lift, high curl, leg press, and upright row. To clarify, assisted pull-ups involved shoulder frontal plane adduction and elbow flexion, and were performed using a machine that applies a selected force under the body when necessary. High curls involved elbow flexion and shoulder sagittal plane flexion, and required a standing barbell curl through the first half of a normal range of motion followed by a front raise of the shoulders to simulate lifting an object to a high platform. The endurance training was conducted mainly in 80-minute periods, consisting of interval running followed by loaded marching with individually prescribed loads that increased progressively over the 10 weeks. Just as for the Circuits group, the circuit training generally consisted of high-repetition, low-force exercises using all major muscle groups, while sports periods were typically team ball games in a small area.

\section{Testing}

All testing took place in late spring and summer in England, and environmental conditions were not notably different between testing sessions. The loaded march test was performed both before and after training. The loaded march required subjects to complete a $3.2-\mathrm{km}$ (2 mile) flat bitumen course as quickly as possible while carrying a backpack (Bergen) loaded to $25 \mathrm{~kg}$. Subjects wore training shoes and lightweight military clothing and did not carry rifles. Subjects were started individually at 30-second intervals to encourage maximum effort. A separate sample of 9 subjects ( 6 men, 3 women) performed the loaded march test on 2 separate occasions 3 days apart to assess test-retest reliability. Good reliability was shown-there was no significant difference between the 2 trials ( $p=$ 0.18 ), an intraclass correlation of $R=0.99$, and ratio limits of agreement (7) of $0.977 * / 1.087$.

Five additional field tests thought likely to be related to loaded march performance were conducted before training only: fat-free mass, isometric lower back extension strength, isometric upright pull strength, dynamic lift strength, and an incremental shuttle run. Fat-free 


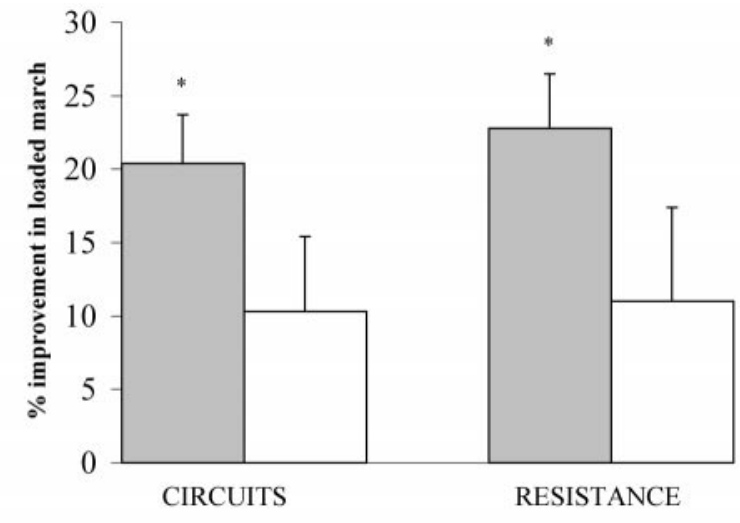

* Significant difference between good and poor responders $(p<0.001)$ within each training mode

Figure 2. Mean $\pm S D$ percentage improvements in loaded march performance for the good (shaded bars) and poor (clear bars) responders to each training mode. *Significant difference between good and poor responders $(p<0.001)$ within each training mode.

mass was estimated using a bioelectrical impedance device (Bodystat 1500, Bodystat, Isle of Man, UK). The maximum force produced during an isometric upright pull at a height of 0.38 meter (an isometric lift) was assessed using a Takei dynamometer, and represents the composite force produced by the muscles involved in hip and knee extension, and shoulder elevation (3). Dynamic lift strength was assessed as the 1RM lift (6) to a height of 1.45 meter, and is related to the dynamic strength of the muscles involved in hip and knee extension, shoulder elevation and flexion, and elbow extension. Isometric lower back extension strength was also assessed using a strain gauge, as described by Poulsen and Jørgensen (8). Aerobic running performance was assessed using a continuous, progressive multistage 20 -meter shuttle run test (2).

\section{Statistical Analyses}

An alpha level of 0.05 was used for all statistical tests. After completion of the training program, Circuits were ranked by percentage improvement in the loaded march, divided into significantly different (1-tailed independent $t$-test, $p \leq 0.001$ ) subgroups of 15 subjects each, and labelled 'good' and 'poor' responders (mean $\pm S D$ improvements of $20.4 \pm 3.3 \%$ and $10.3 \pm 5.1 \%$, respectively). The subgroups were compared for values in the other pretraining tests using 1-tailed independent $t$-tests-the good responders to circuit training in terms of loaded march performance were expected to be stronger but less aerobically fit than the poor responders, and vice-versa. In addition, 3 new variables were created using the ratio of shuttle run endurance against each of the 3 strength tests, and the subgroups were also compared for values in these new variables at the pretraining stage-the good responders were expected to have a lower ratio of endurance performance to strength performance than the poor responders.

A similar procedure was carried out for Resistance (mean $\pm S D$ improvements of $22.8 \pm 3.7 \%$ and $11.0 \pm$ $6.4 \%$ for good and poor responders, respectively) that also provided 2 significantly different $(p \leq 0.001)$ subgroups, each of 10 subjects. For Resistance, it was expected that the good responders would be less strong but more aerobically fit than the poor responders, and vice-versa.

Finally, the relationships between the various pretraining variables and the training-induced percentage change in loaded march performance were examined using 1-tailed Pearson product-moment correlation coefficients.

\section{RESULTS}

The percentage improvements of the good and poor responders within both the Circuits group and the Resistance group are shown in Figure 2.

For Circuits, there were 2 pretraining test variables that discriminated significantly between the good and poor responders to endurance-based training for loaded marching, in addition to the pretraining performance of the loaded march itself. The ratio of shuttle run endurance to upright pull strength, and the ratio of shuttle run endurance to lower back extension strength both discriminated significantly between the good and poor responders (Table 2). In addition, the other ratio variable (the ratio of shuttle run endurance to dynamic lift strength) approached significance ( $p=0.08$, Table 2 ), as did isometric lower back extension strength alone $(p=0.08$, Table 2).

For Resistance, only the pretraining performance of the loaded march itself discriminated significantly between the good and poor responders to resistance-based training for loaded marching (Table 3). Of the other variables, the 3 ratios of shuttle run endurance to strength performance were again the nearest to approaching significance (Table 3).

For the Circuits group, the only significant correlation found was between the training-induced percentage change in loaded march performance and the pretraining loaded march performance $(r=0.47, p<0.005)$. For the

TABle 2. Mean $\pm S D$ pretraining data for the good and poor responders to training (Circuits group).

\begin{tabular}{lccc}
\hline & Good $(n=15)$ & Poor $(n=15)$ & $p$-value \\
\hline Fat-free mass $(\mathrm{kg})$ & $63.1 \pm 6.5$ & $62.2 \pm 7.2$ & 0.37 \\
Lower back extension strength $(N)$ & $1059 \pm 162$ & $963 \pm 192$ & 0.08 \\
38 cm upright pull $(N)$ & $1282 \pm 193$ & $1193 \pm 230$ & 0.13 \\
Dynamic lift strength $(\mathrm{kg})$ & $63.3 \pm 12.2$ & $59.5 \pm 10.6$ & 0.18 \\
20 m shuttle run (s) & $577 \pm 72$ & $615 \pm 94$ & 0.11 \\
20 m shuttle run: lower back extension & $0.56 \pm 0.10$ & $0.67 \pm 0.20$ & $0.03^{*}$ \\
$\quad$ strength & $0.46 \pm 0.08$ & $0.53 \pm 0.12$ & $0.03^{*}$ \\
20 m shuttle run: 38 cm upright pull & $9.41 \pm 2.17$ & $10.64 \pm 2.49$ & 0.08 \\
L m shuttle run: dynamic lift strength & $1506 \pm 146$ & $1336 \pm 92$ & $<0.001^{*}$ \\
Loaded march (s) & & \\
\hline
\end{tabular}

\footnotetext{
* Significant difference between good and poor responders before training.
} 
TABLE 3. Mean $\pm S D$ pretraining data for the good and poor responders to training (Resistance group).

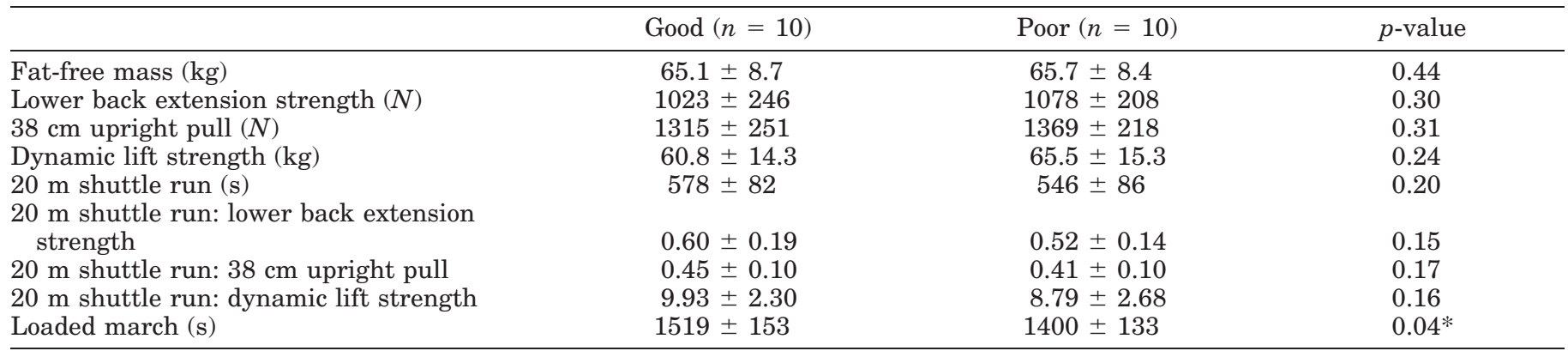

* Significant difference between good and poor responders before training.

Resistance group, significant correlations were found between the training-induced percentage change in loaded march performance and shuttle run endurance $(r=$ $-0.39, p<0.05)$, dynamic lift strength $(r=0.42, p<$ $0.05)$, the ratio of shuttle run endurance to dynamic lift strength $(r=-0.53, p<0.01)$, the ratio of shuttle run endurance to upright pull strength $(r=-0.44, p<0.05)$, the ratio of shuttle run endurance to lower back extension strength $(r=-0.40, p<0.05)$, and the ratio of shuttle run endurance to fat-free mass $(r=-0.39, p<0.05)$.

\section{Discussion}

The results of this study show that simple field tests of strength and endurance can be used in combination to identify those military recruits that would benefit from specific training to improve loaded march performance. Those individuals undergoing British Army basic training (Circuits) who showed a large increase in loaded march performance had significantly lower pretraining ratios of shuttle run endurance to isometric upright pull strength $(0.46$ vs. 0.53$)$ and of shuttle run endurance to lower back extension strength (0.56 vs. 0.67$)$ than those who showed a small increase. Consequently, conducting either a shuttle run and an isometric upright pull, or a shuttle run and an isometric lower back extension, could identify those individuals for whom an endurance-based training program is most appropriate. Given that loaded march performance appears to rely on a combination of strength and endurance $(4,5)$, this is not entirely unexpected. However, these are the first data to identify specific pretraining measures that can be used to prescribe training effectively. For the first time, the intuitive conclusion that endurance-based training should improve loaded march performance to a greater extent for stronger and less aerobically fit individuals than less strong and more aerobically fit individuals, is supported by research evidence.

Additional data are presented which suggest that an alternative training program focused on resistance training would be more appropriate for those individuals who have higher pretraining ratios of shuttle run endurance to isometric upright pull strength, and of shuttle run endurance to lower back extension strength. The data showed the expected tendencies in the opposite direction-less strong and more aerobically fit individuals appeared to improve loaded march performance more than those stronger and less aerobically fit. However, these data from the Resistance group did not reach statistical significance ( $p=0.15-0.17)$.

For both the Circuits and Resistance groups, pretraining performance in the loaded march test itself was also able to discriminate between the good and poor responders to each training mode. This finding was expected, as it is generally accepted that individuals with the greatest capacity to improve a physical performance through training are those with poorer initial performances (1). However, these data are not of great practical value for 2 reasons. First, military recruits are rarely required to perform load carriage early in their basic training program due to the time involved and the risk of injury to untrained individuals. Even more important, however, is the pattern of response to the 2 training programs used in this study. In both groups, the same response was found: those individuals with poorer pretraining performance in the loaded march responded better to training based on either endurance or resistance exercise. Consequently, even though poor responders can be identified using a load carriage test, no specific training program can be prescribed as being particularly appropriate. In contrast, the running endurance-to-isometric strength ratios already described can be used both to identify good and poor responders to a given training mode, and ultimately to prescribe the more appropriate training program for the poor responders.

Only low to moderate relationships were observed between the various pretraining variables and the traininginduced change in loaded march performance. This casts some doubt on the utility of the pretraining test scores in predicting the training response. Given these rather poor relationships, a reasonable area of doubt should be acknowledged when using the pretraining tests identified here to prescribe military training. In other words, for individuals who demonstrate a clear predisposition to strength capability over endurance, endurance-based training should certainly be advised. For individuals who demonstrate a clear predisposition to endurance capability over strength, resistance-based training should be advised. For individuals in whom the ratio of endurance to strength capability appears more balanced, the predictive abilities of the tests used here are probably not sufficient to warrant rigid interpretation. The training actually prescribed may depend more on the local military environment where facilities may partly determine training regimens. It is also interesting to speculate that for individuals who demonstrate no clear predisposition to endurance or strength performance, a combined endurance and resistance training program (which is in fact what the Resistance group undertook) may be most suitable. This suggestion is supported by the raw data, where the improvements for both the good responders $(22.8 \%$ vs. $20.4 \%)$ and the poor responders $(11.0 \%$ vs. $10.3 \%)$ were 
marginally higher for the Resistance group (not statistically significant).

It is important to note that the data presented here are specific to a $3.2-\mathrm{km}$ loaded march with $25 \mathrm{~kg}$. The practical implications are also most relevant to a similar task, and become less relevant as the nature of the task becomes notably different from a 3.2-km loaded march with $25 \mathrm{~kg}$. If a military organization uses a vastly different test of load carriage performance at the end of basic training, then the conclusions drawn here may be inappropriate. Further studies would need to be conducted to provide similar data that are of use in training diagnosis for other load carriage tasks involving greater distance or mass, for example. Furthermore, the approach adopted here is based on the premise that loaded march performance is the sole physical test used at the end of military basic training. Training diagnosis may be more difficult for other important military tasks such as box lifting, where 2 dichotomous variables related to successful performance are not so readily apparent. Of note here are the recent findings that suggest that a training program focused on resistance training is more effective than the traditional British Army basic training at improving performance over a range of common military tasks (13).

Future research, as suggested by Wilson and Murphy (14), could use a similar approach to data collection and analysis as that used here to help provide objective data on which to base rational decisions about the content of physical training programs. It is not our opinion that such data will allow the prescription of training programs without any subjective thought or judgment. However, our research design could be used more often both retrospectively to analyze data already gathered or published, and during new investigations into the efficacy of various training methods. We are of the opinion that the data produced could then be used to shift the role of coaching and the strength and conditioning practitioner towards one based more on science and objectivity.

In conclusion, we were able to show that training diagnosis is possible for load carriage, as some pretraining test values were significantly different between good and poor responders. Some ratios of endurance-to-strength capability were particularly useful in identifying whether endurance or strength-based training is most appropriate for certain individuals. Objective data on which training for load carriage can be prescribed, based on simple pretraining measures, now exists. This work provides a useful tool for military organizations to use in their prescription of appropriate training programs.

\section{Practical Applications}

This study has shown that simple field tests of strength and endurance can be used in combination to identify those military recruits who would benefit from specific training to improve loaded march performance. This training diagnosis, which is conducted before the training actually begins, could be a useful tool for military organizations seeking to improve the effectiveness of training regimens used in basic training. Tests of running endurance and isometric back muscle strength can be conducted in the military environment safely, quickly, and easily. The results of these tests can then be used to create a ratio score of endurance-to-strength capability, and this score used to discriminate between those recruits for whom the British Army basic training program produces large or small improvements in loaded march performance. An alternative training regimen focused on resistance training should be prescribed for those individuals with a high pretraining ratio score of endurance-tostrength capability. Thus, those military recruits likely to show a small improvement in response to the traditional training can be prescribed more effective training in a targeted manner.

\section{REFERENCES}

1. Astrand, P.O., AND K. RodAhl. Textbook of Work Physiology: Physiological Bases of Exercise. Singapore: McGraw-Hill, 1986.

2. Brewer, J., R. RAmsbottom, AND C. Williams. Multistage Fitness Test. Leeds: The National Coaching Foundation, 1988.

3. KNAPIK, J.J., J.A. VoGEL, AND J.E. WRIGHT. Measurement of Isometric Strength in an Upright Pull at $38 \mathrm{~cm}$. US Army Technical Report No. T3/81. Natick, MA: US Army Research Institute of Environmental Medicine, 1981.

4. Kraemer, W.J., J.A. Vogel, J.F. Patton, J.E. Dziados, AND K.L. REYNolds. The Effects of Various Physical Training Programmes on Short Duration, High Intensity Load Bearing Performance and the Army Physical Fitness Test. US Army Technical Report No. T30-87. Natick, MA: US Army Research Institute of Environmental Medicine, 1987.

5. Kraemer, W.J., S.A. Mazzetti, B.C. Nindl, L.A. Gotshalk, J.S. VoleK, J.A. Bush, J.O. MarX, K. DohI, A.L. Gomez, M. Miles, S.J. Fleck, R.U. Newton, AND K. HAKKinen. Effect of resistance training on women's strength/power and occupational performances. Med. Sci. Sports Exerc. 33:1011-1025. 2001.

6. McDaniel, J.W., R.J. Skandis, AND S.W. Madole. Weight Lift Capabilities of Air Force Basic Trainees. US Air Force Technical Report No. 83-0001. Wright Patterson AFB, OH: US Air Force Aerospace Medical Research Laboratory, 1983.

7. Nevill, A.M., AND G. AtKInson. Assessing agreement between measurements recorded on a ratio scale in sports medicine and sports science. Br. J. Sports Med. 31:314-318. 1997.

8. Poulsen, E., AND K. JøRGENSEN. Back muscle strength, lifting, and stooped working postures. Appl. Erg. 2:133-137. 1971.

9. RAyson, M.P. The Development of Physical Selection Procedures. Phase 1: Job Analysis. Contemporary Ergonomics 1998. M.A. Hanson, ed. London. Taylor and Francis, 1998. pp. 393397.

10. SChmidtbleicher, D. Training for power events. In: Strength and Power in Sport. P.V. Komi, ed. Oxford: Blackwell Science, 1992. pp. 381-395.

11. Tidow, G. Aspects of strength training in athletics. New Studies in Athletics 1:93-110. 1990.

12. Williams, A.G., M.P. RAYson, AND D.A. JonEs. Effects of basic training on material handling ability and physical fitness of British Army recruits. Ergonomics 42:1114-1124. 1999.

13. Williams, A.G., M.P. Rayson, AND D.A. Jones. Resistance training and the enhancement of the gains in material-handling ability and physical fitness of British Army recruits during basic training. Ergonomics 45:267-279. 2002.

14. WILSON, G.J., AND A.J. MuRPHY. Strength diagnosis: the use of test data to determine specific strength training. J. Sports Sci. 14:167-173. 1996.

\section{Acknowledgments}

This work has been carried out with the support of the Ministry of Defence. The authors specifically wish to thank the training staff and recruits of the Army Training Regiment at Winchester, Sir John Moore Barracks, Winchester, UK.

This research was conducted at British Army training establishments.

Address correspondence to Dr. Alun G. Williams, a.g. williams@mmu.ac.uk. 